\title{
Infrared thermography for shear stress field measurements in flows
}

\author{
M. Malerba, J. Pirisinu, G. L. Rossi \\ Dipartimento di Ingegneria Industriale - Università di Perugia \\ Via Duranti, 1 - I 06125 PERUGIA - ITALY
}

Keywords: boundary layer, infrared thermography applications, subsonic flows, passive technique, shear stress measurement

In thermo-fluid-dynamics, it is known the important role played by boundary layer. Therefore, the present paper wants to dwell on the possibility to enlarge the application of infrared measurement technique, in particular for subsonic flows analysis. Subsonic flows point out important problems of measurement for the low energy content. In order to overcome this drawback, a high thermal resolution camera has been chosen and used. Moreover it has been experimentally characterized in order to define its modulation transfer function and to provide a tool for data restoration. Tests have been carried out in a subsonic no-cooled closed circuit wind tunnel. That is, during the start-up of facility, a natural air heating is produced by exchange with fan. However, the latter is not localized and equal to few degrees during the whole run. In this way, problems own of active technique are avoided and measurement can be performed in subsonic flows in a way called passive technique. A new theoretical model has also been defined to correlate superficial body temperature maps to convective coefficient distribution and therefore to shear stress field. It has been pointed out for semi-infinite slab in thermal transient conditions and for a continuous convective exchange between air and body. As test case of the proposed measurement methodology a Fokker airfoil was used. Results are presented in this paper for zero angle of attach.

In all aerodynamic aspects, the boundary layer plays a fundamental role. It affects the performances of each body immersed in a fluid in terms of drag and lift. Firstly boundary layer inspection was performed in supersonic vehicle optimization. Later, the growth of fuel prizes moved the interest towards new aspects as the possibility to enhance performances for a power plant or to reduce the flight fuel consumption. Nowadays, the horizons are changed and widened again and some of the aims could be represented by a faster race car or less time for travelling in train. In order to set up an experimental tool, able to analyse the boundary layer, many efforts have been done and some advanced measurements techniques have been developed. Possible experimental chains make use of traditional or advanced methods.

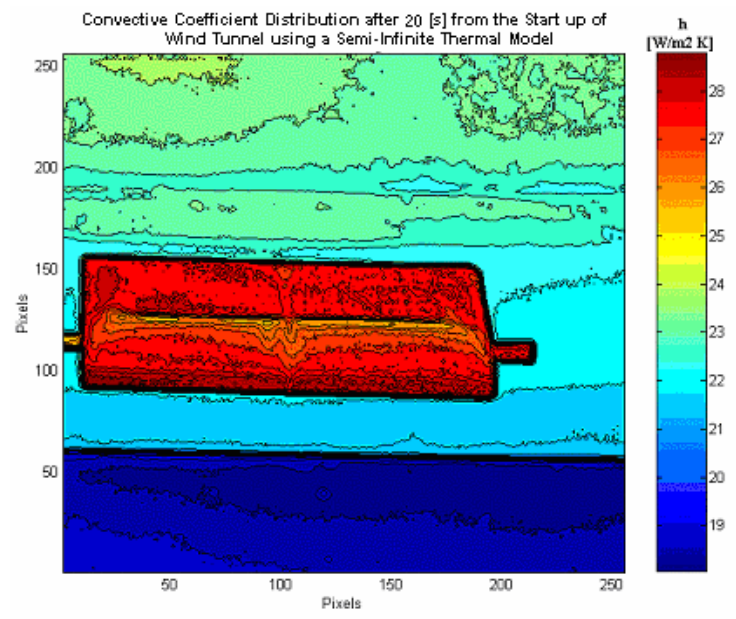




\section{Figure 1: A typical convective coefficient map obtained}

The formers, as with Stanton tubes, are very intrusive and they point out only punctual information. Moreover, their application in turbulent fields is quite inaccurate and it does not produce reliable data [7]. Otherwise, advanced techniques make use of pressure sensitive paints (PSP) [8], temperature sensitive paints (TSP) [8], shear sensitive liquid crystals (SSLC) [9] or oil film interferometry [10]. They are non-intrusive and carry out bi-dimensional maps, but they are very time-consuming and difficult to be performed outside a controlled ambient, as in a laboratory. Since mid-sixties, the use of infrared thermography has started too. Primarily, this technique was used in space missions. Indeed it was fundamental to protect the space shuttle in the re-entry phase. Therefore, special materials were tested to verify their capability to resist to these mechanical and thermal stresses. Later, it became to be wider and wider applied in all the aforementioned sectors. Nowadays it is one of the most advanced measurements and fuses the positive aspects of traditional and advanced measurements; indeed it could be set in hostile ambient, if properly employed, no time-consuming and it performs a non-intrusive measurement of thermal surface distribution, caused by interaction between fluid and body [4]. These maps get information about boundary layer and can be reduced in convective coefficient distributions by an ad hoc thermal model or, using the following Reynolds' analogy, in any parameter of interest for aerodynamic aspects.

$$
\frac{\mathrm{Nu}_{\mathrm{x}}}{\operatorname{Re}_{\mathrm{x}} \operatorname{Pr}}=\frac{\mathrm{C}_{\mathrm{fx}}}{2}
$$

In particular, as described in [6], assuming $\operatorname{Pr}=1$, eq. $(\mathrm{X})$ gives rise to the following linear relationship between convective and skin friction coefficients.

$$
\mathrm{C}_{\mathrm{fx}}=2 \cdot \frac{\mathrm{v} \cdot \mathbf{h}_{\mathrm{x}}}{\mathrm{c} \cdot \mathrm{k}}
$$

An example of results obtained is given in fig 1. In the paper detail of the measurement and processing techniques used to obtain this kind of results are given. 


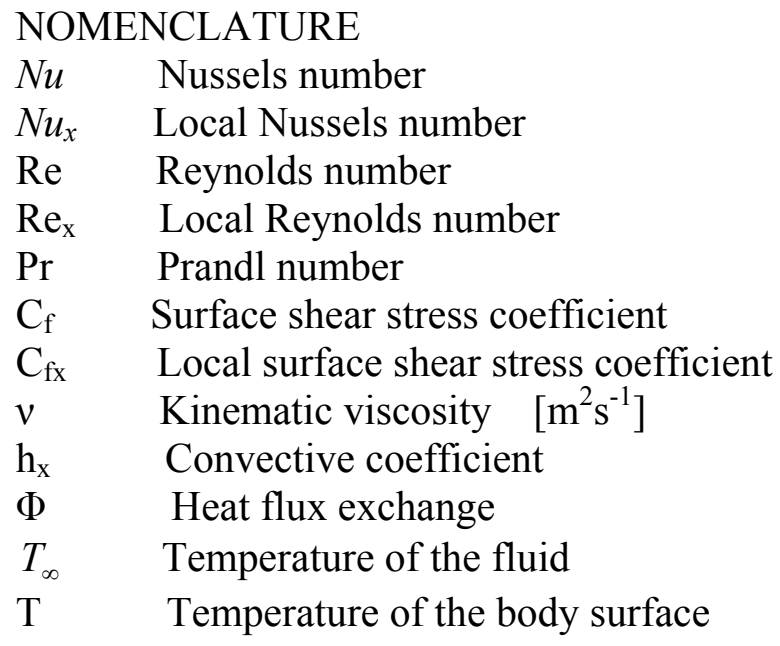

\section{INTRODUCTION}

As well known boundary layer plays a fundamental role on the analysis of fluid dynamic phenomena and on interaction between solid bodies and surrounding fluid flow too. This reason has moved many researchers to identify new measurement techniques to obtain suitable informations and improve a fast investigation of body aerodynamics. Reynold analogy introduce the possibility to use high sensitivity thermographic systems in order to analyse the distribution of shear stress on the surface of solid bodies immersed in fluid knowing the temperature of the examined body surfaces. Reynold's analogy can be expressed with the following relation [1]:

$$
\frac{N u_{x}}{\operatorname{Re}_{x}^{*} \operatorname{Pr}}=\frac{C_{f x}}{2}
$$

where

$$
C_{f x}=2 * \frac{v^{*} h_{x}}{c^{*} k}
$$

In this way aerodynamic drag estimation can be performed by knowing the coefficient of heat exchange $h_{x}$. The main problem to take in account using a thermographic measurement system on dynamic fluid study is the energy associated to the fluid flow. In fact it is correlated with Mach number $[2,3]$. That means that the energy of subsonic flow is not sufficient to produce a remarkable temperature change on the body surface. Usually to overcome this problem the body surface is artificially heated: increasing the heat exchange. This is the so called "active technique". If this technique can not be performed, to increase the energy of heat exchange the fluid flow can be heated (passive technique). In both case the external heating can cause modification of interaction phenomena between solid bodies and the surrounding fluid flow. Anyway the passive technique is to prefer to the active one: in fact it has minimal interaction [4].

\section{MEASURAMENT CHAIN/ PRINCIPLE}

Measurement principle used in this study, is based on the heat exchange that take place between a solid body and the surrounding fluid flow. As well known the total amount of energy taken in account by the heat exchange can be divided in three components: the energy due to the convection, conduction and radiation. Heat transfer between body and fluid is governed by conduction and 
convection phenomena, while energy acquired by thermographic systems is due to the infrared radiation of the body surface (Planck theory). Neglecting conduction phenomenon, the energy associated with convection between body and fluid, can be described as:

$$
\Phi=h_{x}\left(T_{\infty}-T\right)
$$

Heat transfer coefficient $h_{x}$ change if the flow is laminar or turbulent. As before mentioned, in order to monitor boundary layer by IR thermography, it is fundamental the presence of a thermal exchange between model and fluid [5,6]. One of the main aspects is represented by conversion of temperature from static to total values due to the interaction between body and fluid it is immersed in. As consequence, the trend of total temperature will be characterized by a maximum at stagnation point, followed by a first decrement until turbulent area, where it will undertake a local rise. The area of trend inversion is said transition or aerodynamic bubble. Obviously this distribution will condition the thermal exchange between body and fluid. At first glance, it seems that higher superficial temperature will be placed in coincidence of higher fluid recovery temperatures. More in deep, near stagnation, velocity of fluid is very low and heat transfer happens mainly for conduction and; considering the high thermal resistance of air, the heat flux between body and fluid is reduced. However, at the same time, the boundary layer will have its minimum thickness. Globally, the result is usually a maximum of body temperature in the leading edge with a successive linear decreasing. So using a thermographic system it is possible to acquire temperature maps that contain information about boundary layer and they can be reduced in convective coefficient distributions by thermal model or, using the Reynolds' analogy. The value of heat transfer coefficient depends from many factors (i.e. geometry, Prandtl number etc.). It means that is not possible to obtain a general numerical procedure to determine heat transfer coefficient from the superficial temperature map. Usually an easier approach is to consider the body characterized by a low thermal resistance and capacity. Anyway in this work qualitative tests are made in order to understand if it is possible to associate areas with different temperature gradient to different kind of boundary layer. The thermographic system used is a high sensitivity CCD thermocamera, manufactured by Stress Photonics, the Deltatherm 1560 system.

\section{POSSIBLE NEW APPLICATIONS}

High resolution thermographic system opens up new possibility for aerodynamics subsonic testing. In fig 1 is shown a thermal map obtained by testing a racing motorcycle in wind tunnel.

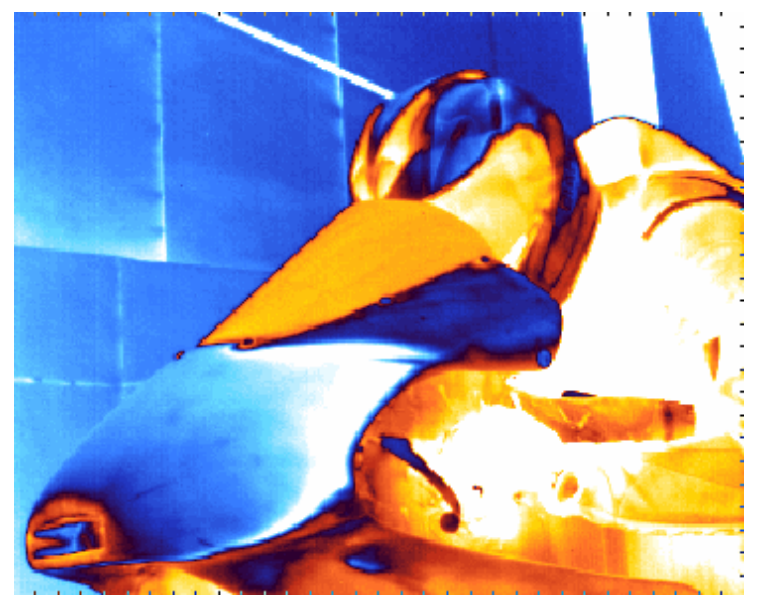

Fig 1: Thermal map of the front of a motorcycle in the wind tunnel. 
Anyway in subsonic flows heat exchange by conduction and convection are both relevant and many disturbing input take places, therefore quantitative measurements are difficult to perform. In this work in order to be more confident with the particular thermographic system used two classical simple cases are tested: a flat plate and a cylinder.

\section{FLAT PLATE}

A flat plate with overall dimension of $1 \times 2 \mathrm{~m}, 40 \mathrm{~mm}$ thick was tested inside a wind tunnel, in a test section of $2 \times 2 \mathrm{~m}$. To avoid infrared reflections and effects of different emissivity values on the flat plate surface, the flat plate was covered with a mat black paint. The flat plate was initially fixed on the test section of the wind tunnel using 0 degrees angle of attack as illustrated in fig 2.

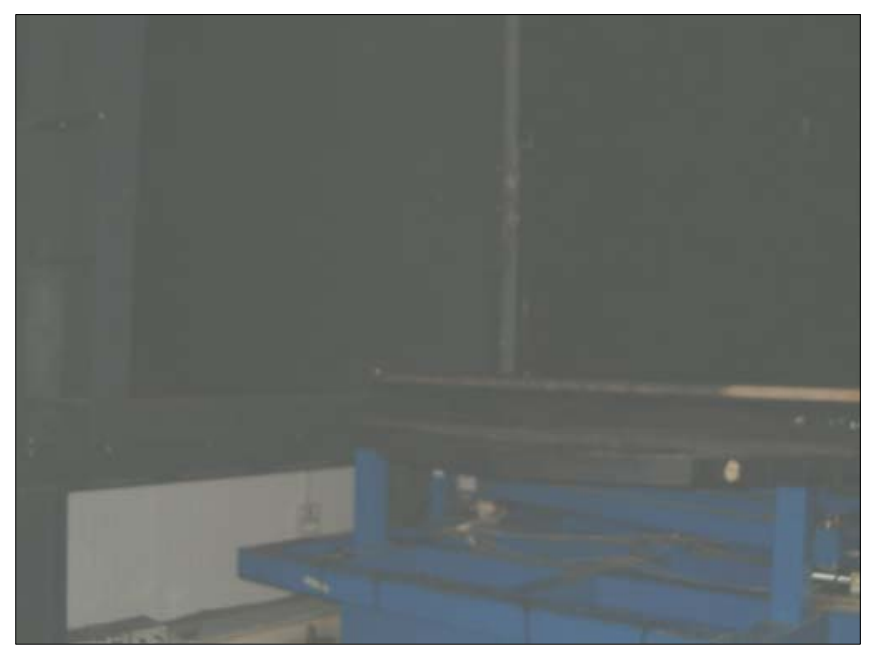

Figure 2 : flat plate in wind tunnel

During the wind tunnel run-up, the air temperature increases because of the dissipation of the fan power occur inside the flow and no air conditioning is used. On the flat plane a warmer flow impact and produce a thermal exchange between flow and body.

In a first test the impact area of the flat plane was characterized by a squared corner. Thermographic results are shown in the following figures that represent temperature distribution on the surface of the flat plate, acquired at two different instant.

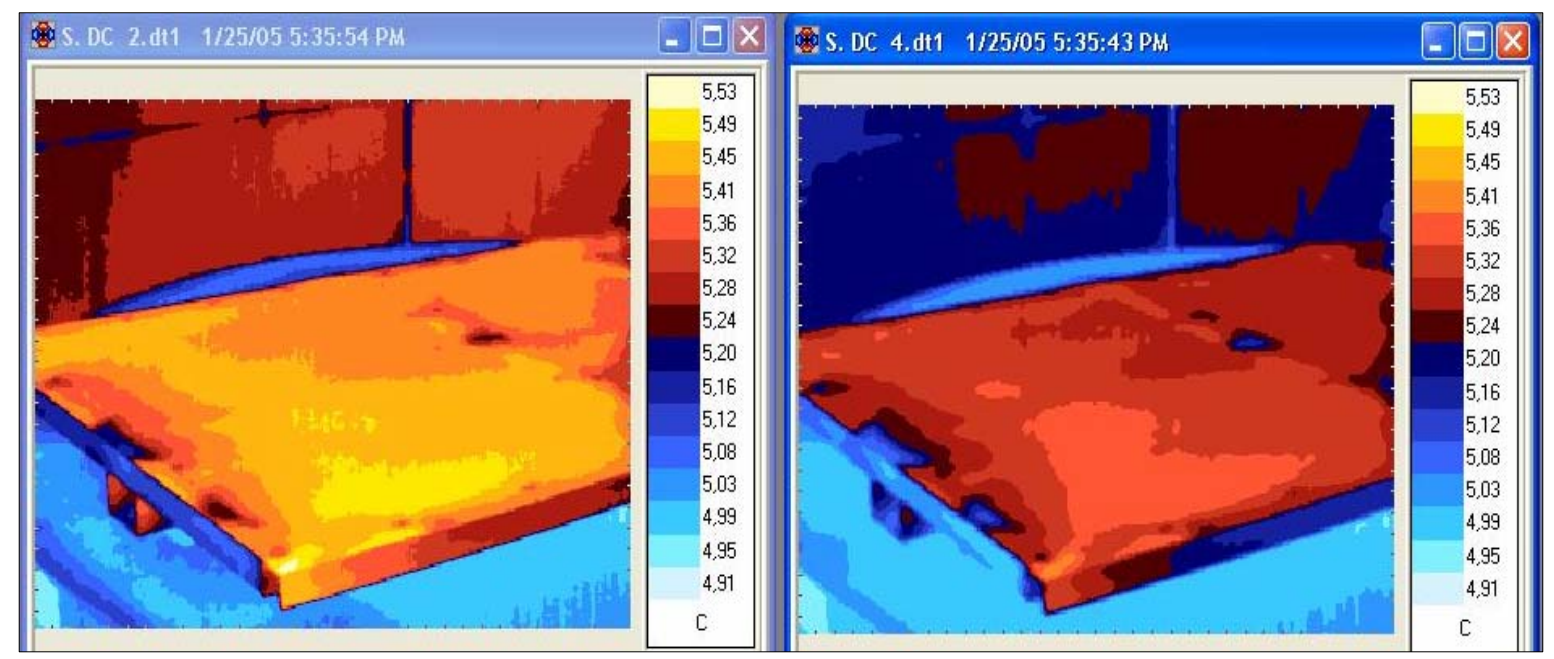

Figure 3: thermographic images acquired in two different instant 
To better understand sense of acquired data a control line is used to monitor temperature distribution (figure 4). Fig. 5 shows the temperature profile along this line. It is characterized by a rapid temperature increase just behind the impact zone than it slowly decreases. That mean a first prominent heat exchange, a diminution and then a new increment. By observing this temperature distribution and knowing that heat exchange is bigger when flow is attached or a turbulent transition occurs, it is possible to assume that flow after the impact zone detaches from the plate surface. After a while it reattaches and temperature continues to increase also due to the temperature rising during the test. The decrease of temperature marked in figure is due to the start of detaches of the flow stream. The following temperature raising is due to the strong heat exchange that characterizes the transition zone.

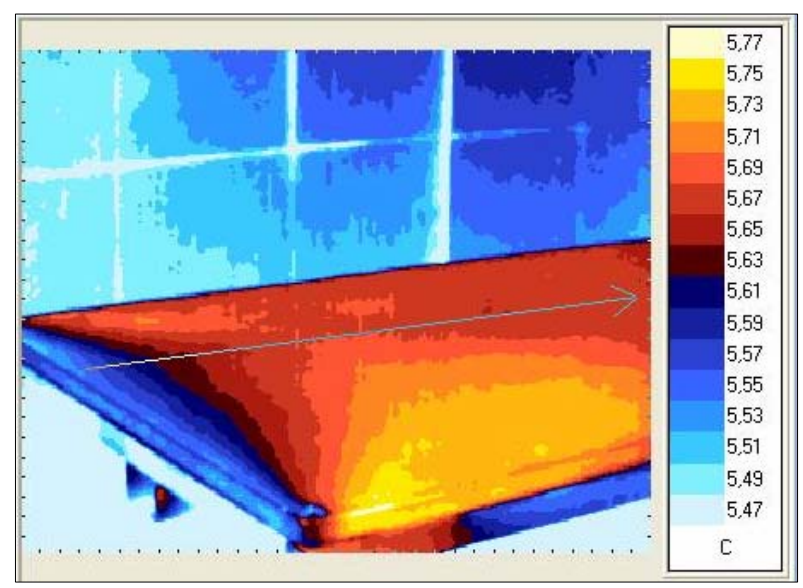

Figure 4: interrogation line

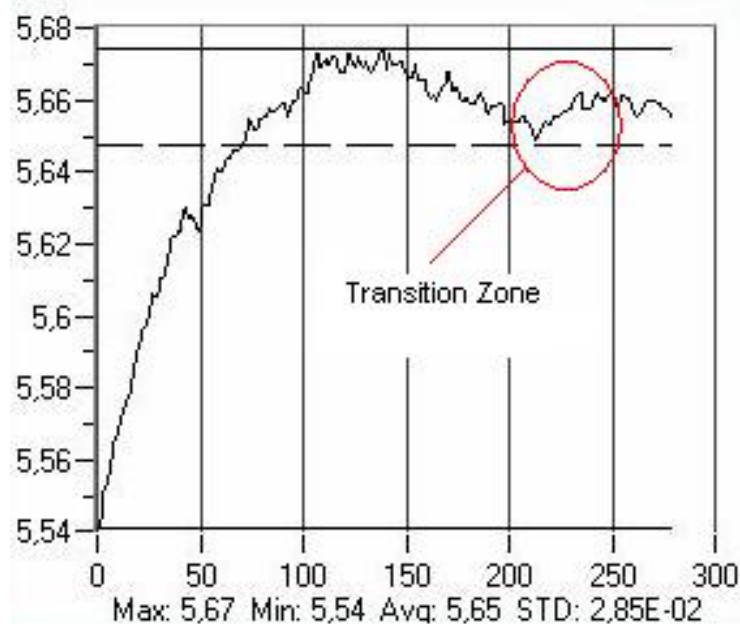

Figure 5: temperature curse along the line

In a second test case, to reduce detach phenomenon and have a qualitative validation of this hypothesis, the corner of the edge of attack is smoothed. The following figure shows the smoothing edge. It is characterized by two different zones. The first one on the left of figure 6 , has a junction radius of $30 \mathrm{~mm}$, the other side has an $8 \mathrm{~mm}$.

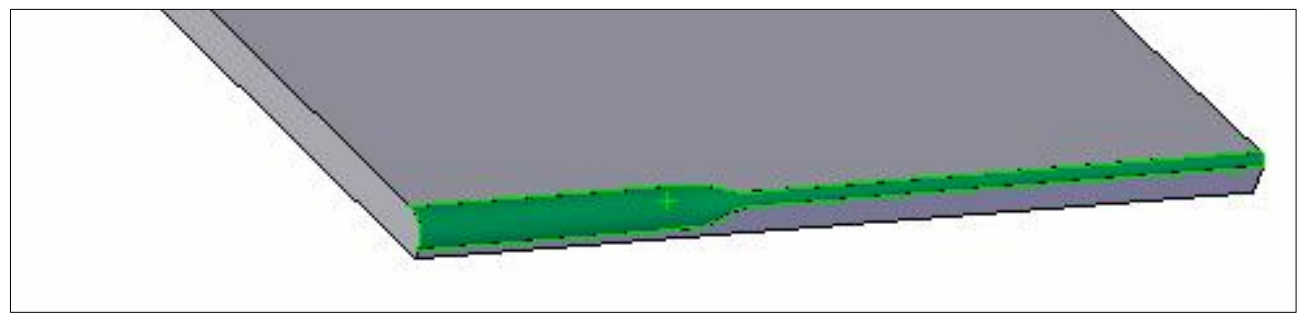

Figure 6: impact zone fillet

Monitoring temperature after smoothed the temperature pattern on the surface of the flat plate is characterized by a different course. Two control lines are signed in figure 7 and 8 . On the right part of each figure is shown the temperature state. Temperature distribution is confirmed in its general purpose but each phenomenon occurs in different zone. It is important to observe that distance of the transition zone from the impact is closer to the edge with a fillet of $8 \mathrm{~mm}$, than the case of $30 \mathrm{~mm}$ 
fillet, and the temperature gradient is stronger. It means that in the case of $8 \mathrm{~mm}$ radius, transition occurs first than in the other case.
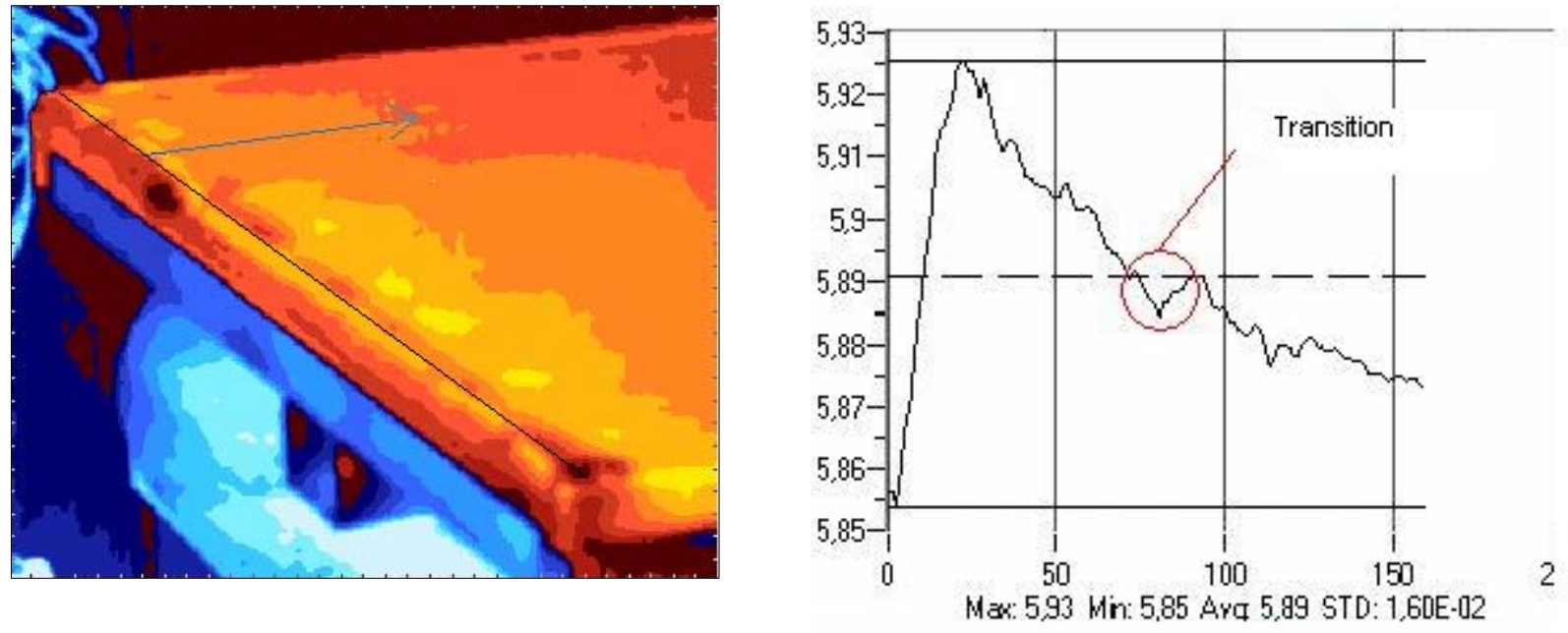

Figure 7: temperature pattern along from the $30 \mathrm{~mm}$ fillet.
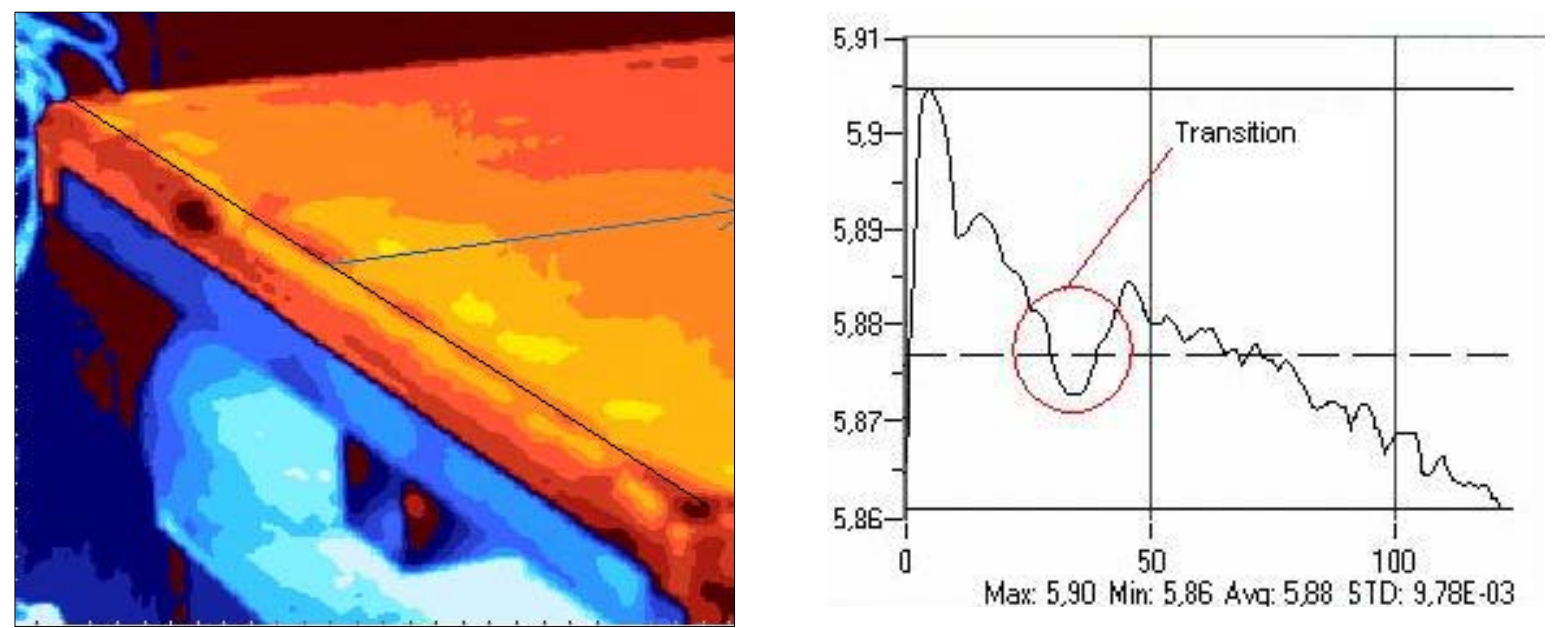

Figure 8: temperature pattern along from the $8 \mathrm{~mm}$ fillet.

\section{CYLINDER}

In order to analyse the possible application of the measurement and testing technique another test case is used: a cylinder.

As well known on a cylinder surface the flow separation may result from a pressure increase or from a curvature effect. At a given location at the wall, the boundary-layer velocity distribution $u(y)$ has a positive slope $\partial \mathrm{u} / \partial \mathrm{y}$. If the pressure increase in the $\mathrm{x}$-direction, an adverse pressure gradient 
$\partial \mathrm{u} / \partial \mathrm{y}>0$ will decrease with $\mathrm{x}$. If the adverse pressure gradient persists, at some point the gradient $(\partial \mathrm{u} / \partial \mathrm{y})$ will be zero and the flow will separate from the boundary. Downstream of separation a back flow exists. A separation streamline divides the main flow from the recirculating flow and fluid does not pass from the main flow into the separated region. However, turbulent mixing of fluid does occur between the two regions. The typical flow on a circular cylinder of infinite length is shown in fig 8:

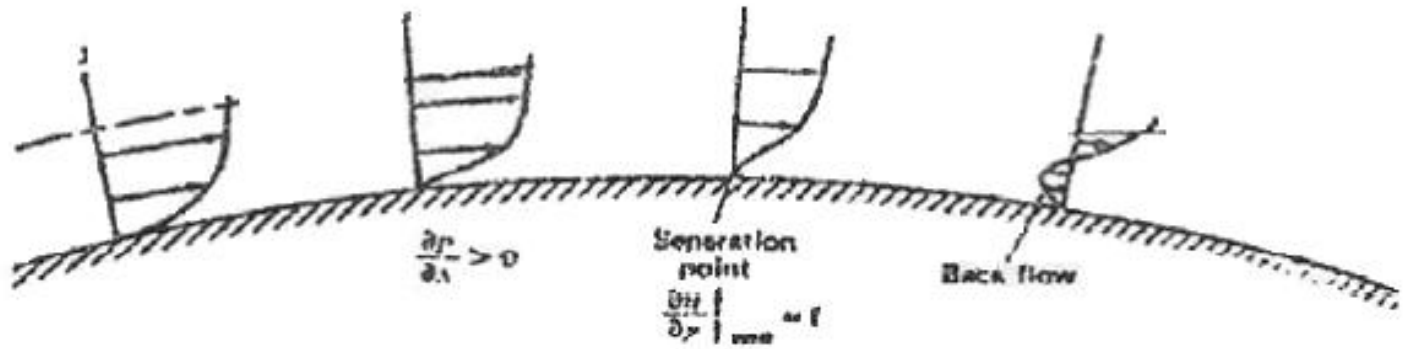

Figure 8: Flow velocity pattern on a transversal section of a cylinder.

The flow is completely laminar until turbulence is generated in the vortex-wake region. The wake then becomes turbulent and the boundary layer too. It occurs just before the separation point. The separation point on the cylinder is not stationary but oscillates around an average location. Initially a vortex is generated and the separation point moves from its origin point to another, is then 'shed' from the cylinder and the separation suddenly moves back. In this zone temperature increase and can be observed whit thermal camera.

The test case used is a PVC cylinder with $460 \mathrm{~mm}$ of radius, $3000 \mathrm{~mm}$ length and $5 \mathrm{~mm}$ of thickness, fixed transversally to the flow, as shown in figure 9.

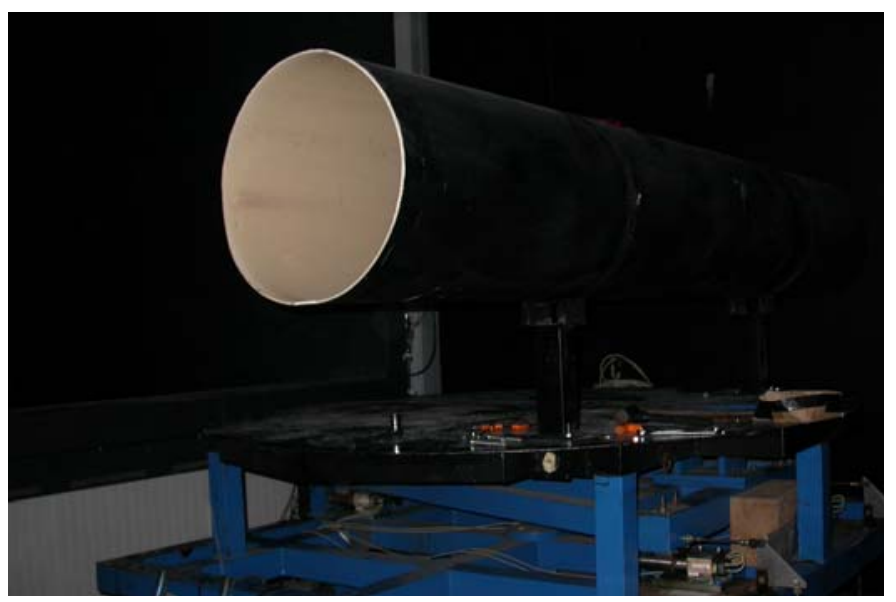

Figure 9: Cylinder installed in the wind tunnel

In the figure 10 thermal state is shown. 

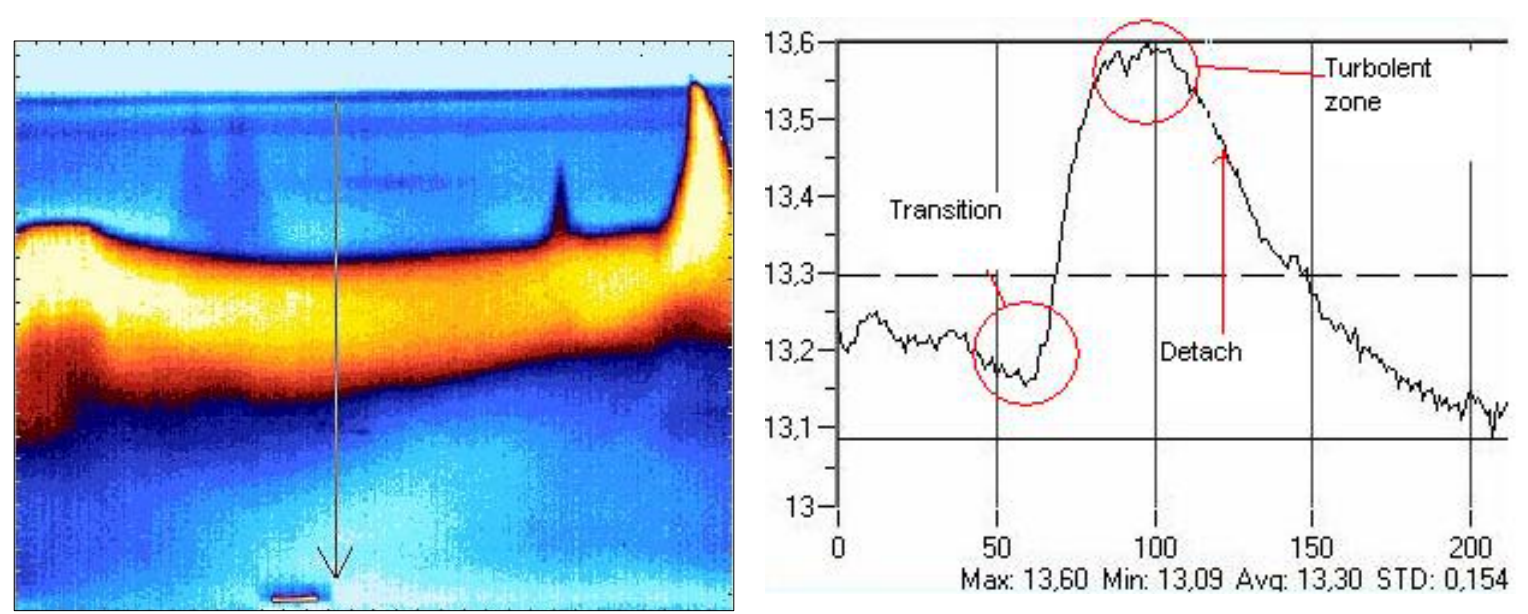

Figure 10: boundary layer transition.

The graph (fig $10 \mathrm{~b}$ ) represents temperature along the line tracked on fig $10 \mathrm{a}$. By the temperature pattern is possible to define a first zone characterized by a slow heat exchange (laminar boundary layer), till a temperature decrease followed by a heavy increase. This zone characterized by the changing of the temperature gradient sign is the transition zone (Fig 10 b).

To have a better known of phenomenon, other tests are performed. It's known that a control of boundary layer can be done. The point of separation can be controlled with active technique. Removing air whit some suction slot on surface or making the fluid turbulent using vortex generator can perturb this zone. Alternatively a high-speed inject of fluid on the separation zone can be done. All these techniques serve to increase the momentum in the boundary layer delaying separation.

To perturb detachment zone the technique of "air injection" is used. To reach necessary speed of air inject, an intake on the impact front side of the cylinder is done (Fig11). The temperature map obtained in this configuration is shown in figure 12.

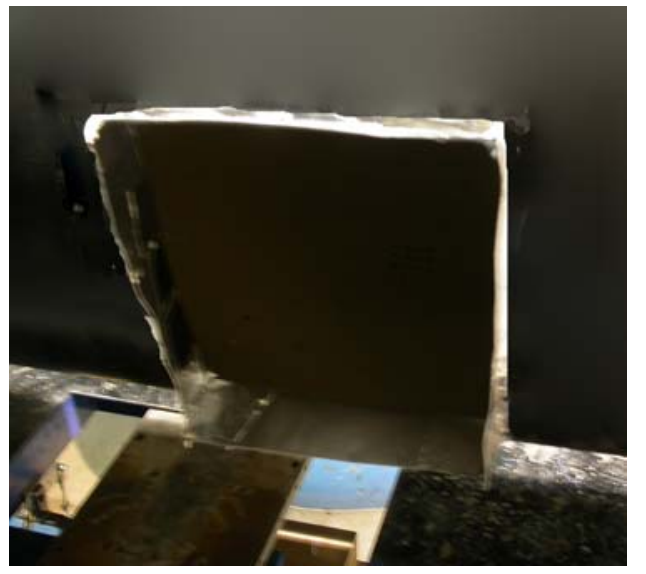

Figure 11: Flow intake in the Cylinder. distribution close to

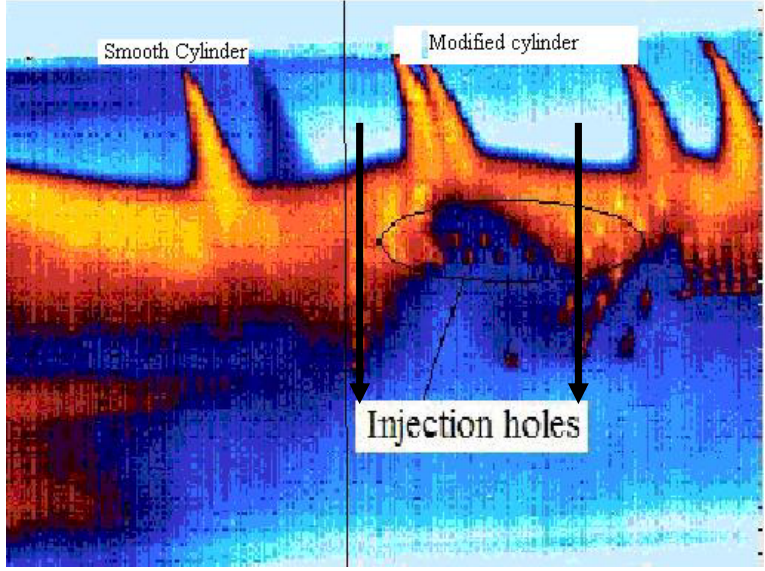

Figure 12: temperature

the hole of air injection.

Figure 12 shows a comparison between smooth cylinder and the one whit inject Comparing temperature profile along lines plotted on the part of the cylinder interested by the air inject (fig 13) to the one not interested by air inject (fig 14) it is possible to 
notice the difference of the length of turbulent one. In fact like previous cases zones interested by high temperature represent turbulent transition zones.

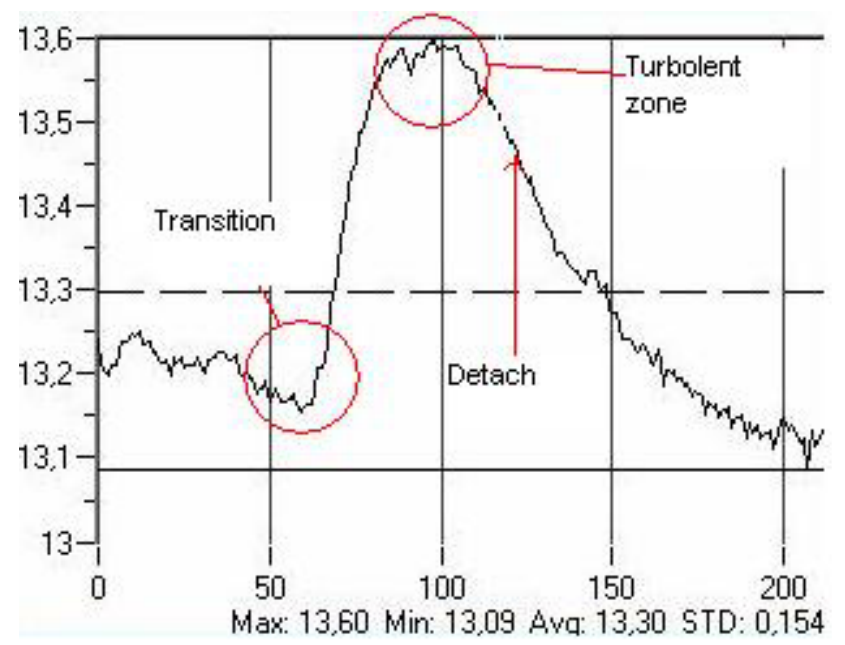

Figure 13: temperature pattern on the air injection

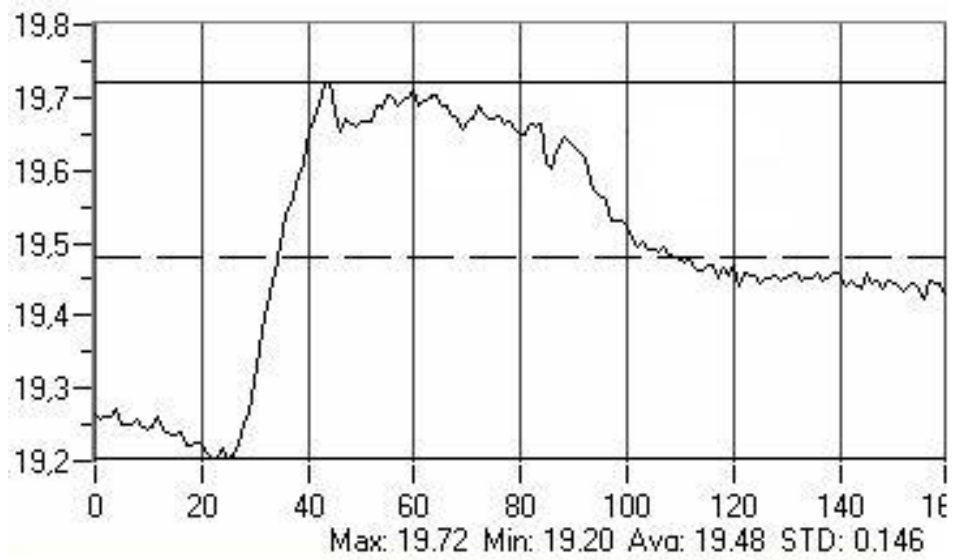

Figure 14: temperature pattern of a zone no interested by air injection

\section{CONCLUSION}

In this work an high sensitivity thermographic system is used to perform a qualitative inspection of boundary layer in low speed flow using Reynold's analogy. To accelerate flow and increase heat exchange between body and flow, the natural heating due to drag phenomena on the circulating air of a closed circuit wind tunnel was used. Two interesting easy cases have been studied, addressing possible aspects that can be detected and optimized with the proposed technique. Also the technique of the control of boundary layer whit high speed air inject is here used. The zone of air injection is the one interested by fluid flown transition. Results explained on this paper, demonstrate that the measurement technique can be used to obtain a fast qualitative evaluation of boundary layer. and realize a fast inspection or a comparison between different solutions, reducing the experimental test duration. Another aspect of interest is that CFD numerical code have the problem of boundary layer modelling, so it should be very useful to integrate a numerical model with the experimental data that can be obtained with the technique here described.

\section{REFERENCES}

[1] F. Kreith, "Principi di Trasmissione del Calore",Liguori Editore,1988. 
[2] T. Astarita, G. Cardone, G. M. Carlomagno, C. Meola,"A survey on Infrared Thermography for Convective Heat Transfer Measurement", Optics \& Laser Technology, Elsevier Science 200 I .

[3] G. M. Carlomagno, L. De Luca, "Infrared Thermography for Flow Visualization and eat Transfer Measurement".

[4] E. Gartenberg, A. S. Roberts Jr., "Twenty-Five Years of Aerodynamics Research with Infrared Imaging", Joumal of Aircraft,Yol.29, No2, March-April 1992.

[5] D. L. Balageas, A-M. Bouchardy, "Application of Infrared Termography in Fluid mechanics", Lecture Series on "Measurement Techniques", Von Karman lnstitute for Fluid Dynamics, 1993-05.

[6] E. Gartenberg, A. S. Roberts Jr., G. J. McRee,"Infrared Imaging and Tufts Studies of Boundary Layer Flow Regimes on a NACA 0012 Airfoil", CH2762-3189/0000- 0168, 1989 IEEE

[7] "Fluid Mechanics Measurement", R. J. Goldstein, 1983, Hempisphere Publishing Corporation

[8] J. Sullivan, "Temperature and Pressure Sensitive Paint", Lecture Series on "Advanced Measurement Techniques", Von Karman Institute of Fluid Dynamics, 2001-01

[9] D.C. Reda, M. C. Wilder "The Shear Stress Liquid Crystal Coating Method: Measurement of Continuous Surface Shear Stress Vector Distributions", Lecture Series on "Advanced Measurement Techniques", Von Karman Institute of Fluid Dynamics, 2001-01

[10] D. M. Driver, “Oil Film Interferometry Technique for Measuring Skin-Friction”, Lecture Series on "Advanced Measurement Techniques", Von Karman Institute of Fluid Dynamics, 200101 